\title{
Reconstruction of a completely obstructed rectosigmoid vaginal introitus in a transgender woman
}

Tae Hyeon Lee, Jung Soo Yoon, Hee Chang Ahn

Department of Plastic and Reconstructive Surgery, Hanyang University College of Medicine, Seoul, Korea
This article was presented at the 8th Research \& Reconstruction Forum on April 20, 2018, in Jeonju, Korea.
Rectosigmoid vaginoplasty is a frequently used surgical method in male-to-female sex reassignment surgery. However, severe side effects, such as sepsis, may occur owing to stricture, mucorrhea, and rectovaginal fistula. Herein, we present the case of a 29-yearold patient who underwent complete reconstruction of an obstructed rectosigmoid vaginal orifice. The patient had undergone male-to-female sex reassignment surgery with rectosigmoid vaginoplasty 8 years previously. The vaginal introitus was completely obstructed, and mucous secretions of the rectum remained in the blind pouch. Therefore, she developed several complications, including panperitonitis, sepsis, and repeated obstruction. We performed complete resection of the scar and reconstructed the orifice of the neovagina using a pudendal thigh island flap. The patient's lifethreatening condition subsided with the successful release of the vaginal orifice. Vaginal orifice stricture is a frequent complication of rectosigmoid vaginoplasty. A pudendal thigh flap can be used to effectively and safely release this stricture and reconstruct the neo-orifice of the rectosigmoid vagina without recurrence.

Keywords Sex reassignment procedures / Vagina / Stricture / Surgical flap

\section{INTRODUCTION}

Patients with vaginal agenesis, gender identity disorder, and gynecologic neoplasms or trauma require vaginal reconstructions to restore their sexual function and aesthetic "shape" [1-3]. Various methods have been developed for successful reconstruction, such as the use of split-thickness and full-thickness skin grafts, buccal mucosal grafts, penile skin inversion flaps, and penoscrotal inversion flaps and rectosigmoid vaginoplasty [1-3]. Of these techniques, rectosigmoid vaginoplasty is a frequently used surgical method in male-tofemale sex reassignment surgery [4]. This procedure has the following advantages over other conventional surgical procedures: a

Received: Mar 4, 2020 Revised: Mar 12, 2020 Accepted: Mar 16, 2020 Correspondence: Hee Chang Ahn Department of Plastic and Reconstructive Surgery, Hanyang University College of Medicine, 222-1 Wangsimni-ro, Seongdong-gu, Seoul 04763, Korea

Tel: +82-2-2290 8560, Fax: +82-2-2295-7671, E-mail: ahnhc@hanyang.ac.kr

Copyright @ 2020 The Korean Society for Aesthetic Plastic Surgery.

This is an Open Access article distributed under the terms of the Creative Commons Attribution Non-Commercial License (https://creativecommons.org/licenses/by-nc/4.0/) which permits unrestricted non-commercial use, distribution, and reproduction in any medium, provided the original work is properly cited. $\quad$ www.e-aaps.org more natural appearance of the neovagina, increased vault length, erogenous sensibility, adequate lubrication from the rectosigmoid flap, and less potential for contraction and vaginal dilator use [5]. Nonetheless, stenosis/stricture of the rectosigmoid neovagina still has a high incidence, occurring in $22.5 \%$ of patients [5]. Stenosis causes fluid collection inside the rectosigmoid neovagina, which may lead to life-threatening septic conditions, such as peritonitis.

The management of complications, such as stenosis/stricture, after rectosigmoid vaginoplasty may be challenging. Data on the therapeutic outcomes of various complications are limited; furthermore, there is currently no widely accepted protocol or guideline [6]. Herein, we report a case of complete reconstruction of a recurring stricture and obstruction of the neovaginal introitus with the use of a pudendal thigh island flap in a transgender woman who had undergone male-to-female sex reassignment surgery with rectosigmoid vaginoplasty.

\section{CASE REPORT}

A 29-year-old patient had undergone male-to-female sex reassignment surgery with rectosigmoid vaginoplasty 8 years prior. Three years later, panperitonitis developed, and surgery was performed 
several times to manage the recurrent stricture and obstruction. Approximately 3 months before visiting our hospital, fluid collected within the neovaginal lumen and sepsis developed. Episiotomy was immediately performed to drain the internal pus from the obstructed neovaginal introitus. The patient's general condition recovered after sepsis care involving surgery and antibiotic adminis-

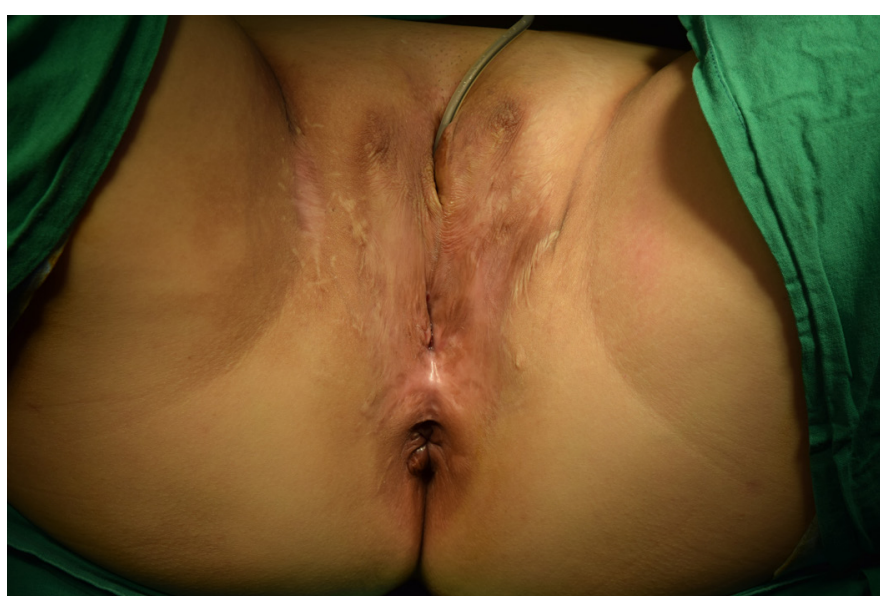

Fig. 1. Complete obstruction of the vaginal introitus after rectosigmoid colon transfer in a transgender woman (preoperative).

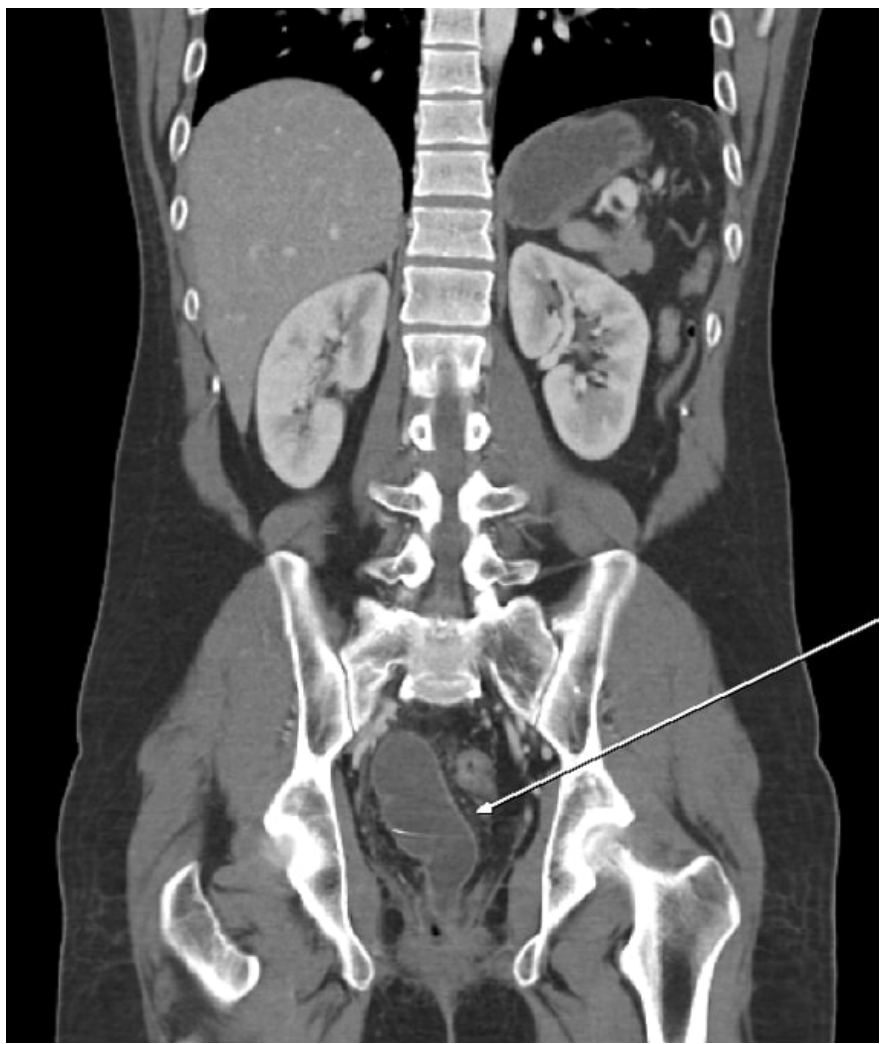

Fig. 2. Abdominal computed tomography (CT) shows fluid collection in the obstructed neovaginal pouch owing to obstruction of the vaginal orifice (white arrow) (preoperative CT). tration. She then visited our clinic to resolve the recurrence of the stricture and its associated obstruction.

The patient was a heavy smoker (40 cigarettes a day) and a heavy drinker ( 5 times a week) but was relatively healthy with no specific disease other than the above-mentioned history of panperitonitis and sepsis. The neovaginal introitus was completely occluded, such that neither a finger nor a Hegar dilator could enter (Fig. 1). Mucous secretions from the rectum remained in the blind pouch (Fig. 2).

Multiple scars were observed around the perineum. A Foley catheter was inserted, and a urologist confirmed that the urethra and bladder were intact. The opening of the neovagina was constricted to a pin-point size. The scar tissue near the fibrotic introitus and the granulation tissue inside the neovagina were removed, and fur-

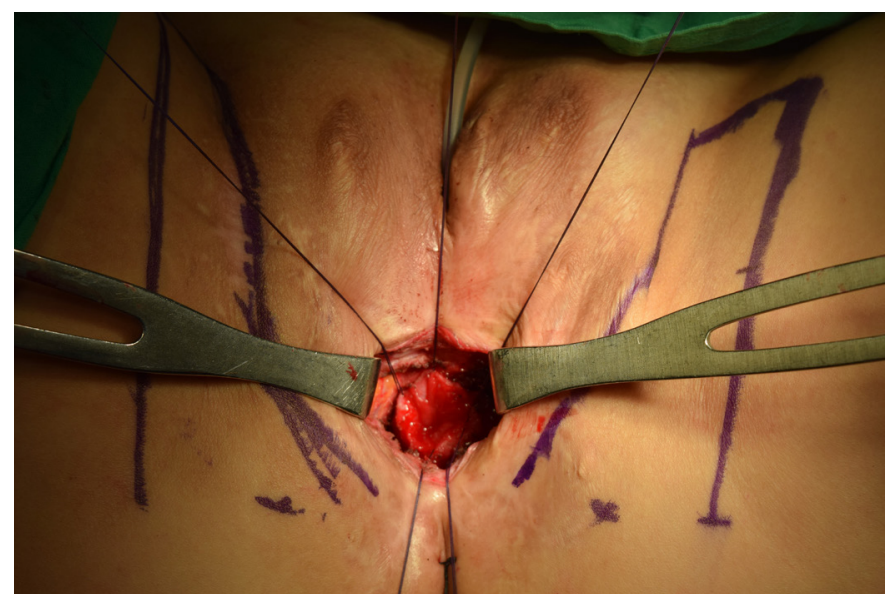

Fig. 3. The edge of the colonic wall of the rectosigmoid colon was dissected and labeled using a silk suture. A pudendal thigh island flap was designed from both sides of the perineum near the defect; the left-side flap was used for the vaginal introitus (intraoperative 1).

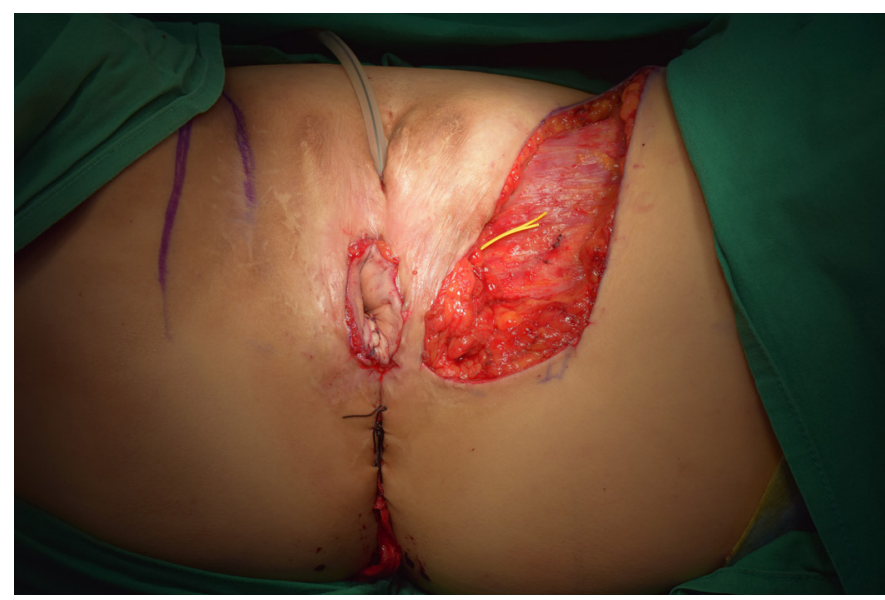

Fig. 4. A $5 \times 8-\mathrm{cm}$ flap was rotated carefully after elevation. The flap had a conduit shape; one side of the flap was sutured to the edge of the inner colonic mucosal layer that had been labeled previously and the other side of the flap to the pudendal skin (intraoperative 2). 

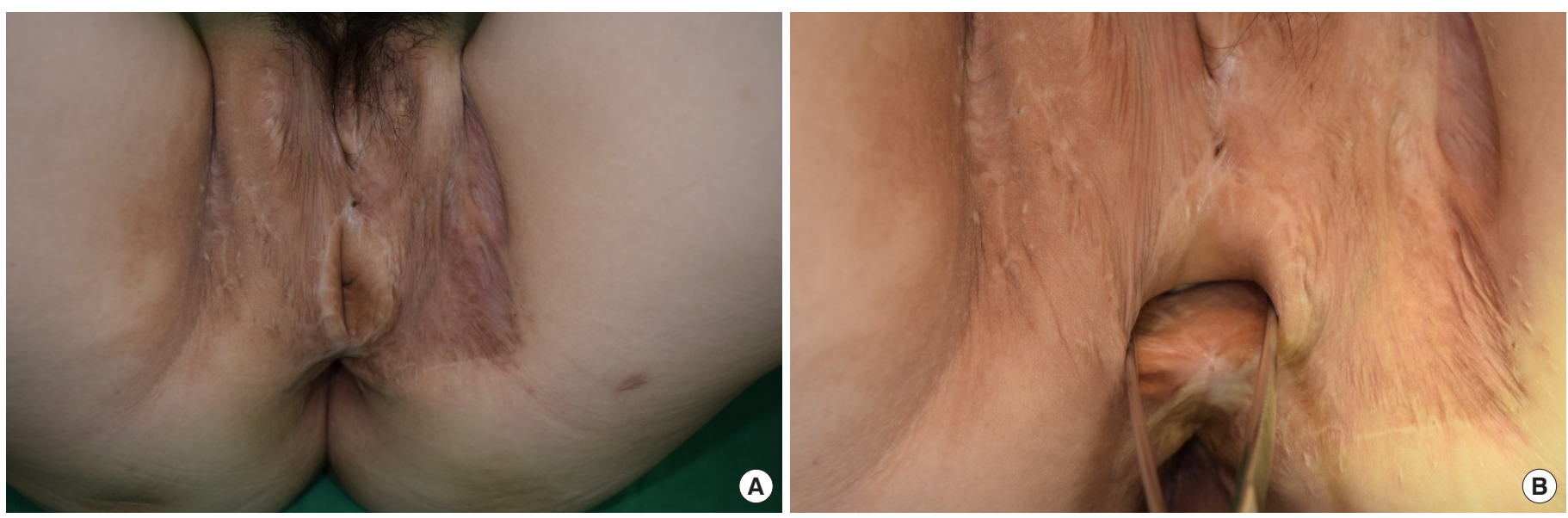

Fig. 5. (A, B) Complete release of the stricture and reconstructed neo-introitus of the rectosigmoid vagina at postoperative 14 months.

ther dissection was performed to define the edge of the colonic wall of the rectosigmoid neovagina, which was labeled using a silk suture (Fig. 3). The fibrotic and granulation tissues of the distal part were completely removed from the colonic edge, and sufficient space for the neo-introitus was secured. The area of the circumferential defect was about $5 \times 8 \mathrm{~cm}$. A pudendal thigh island flap was designed from the left perineum of the defect. The flap measured $5 \times 8 \mathrm{~cm}$ and was rotated carefully after elevation. In order to allow the flap to rotate smoothly towards the defect, the terminal branches of the superficial perineal artery of the flap base were sufficiently dissected and the distal two-thirds of the flap was elevated in a thin manner by dissecting up to the level of Colle's fascia. It had a conduit shape; one end was sutured to the edge of the inner colonic mucosal layer, and the other end to the outer skin layer (Fig. 4). The donor site was closed primarily. One Hemovac drain was inserted into the donor site, and a Foley catheter was inserted into the neovagina to prevent stricture.

On the 9th day after surgery, the neovaginal Foley catheter and Hemovac drain were removed. A small dehiscence was observed on the inferior side of the flap 10 days after surgery; with the aid of daily dressing and sitz bath treatment, most of the stitches were removed on the 15th day. She was discharged on the 23rd day.

She was followed up until 14 months postoperatively and was living a healthy life, free from the life-threatening condition. As of the last follow-up, no wound problems, such as necrosis, fistula, stricture, or obstruction, were observed, and foul odor, dyspareunia, vaginal bleeding, or urinary symptoms did not occur. Even without the use of any dilator devices, the vaginal introitus could be accessed. No stricture/obstruction was present (Fig. 5).

\section{DISCUSSION}

In transgender patients, rectosigmoid vaginoplasty is a frequently used male-to-female sex reassignment procedure. Recently, this procedure has been used more frequently because of its advantages, such as a more natural appearance of the neovagina, increased vault length, erogenous sensibility, adequate lubrication from the rectosigmoid flap, and less potential for contraction and vaginal dilator use [5]. However, severe recurrent stricture/obstruction may occur, as in our case, and once it occurs, its management is very challenging.

In this patient, panperitonitis and sepsis occurred owing to recurrent stricture and obstruction after rectosigmoid vaginoplasty. Several surgical procedures were performed, but did not effectively release the stricture.

There are several reasons why these strictures occur after rectosigmoid vaginoplasty. We suggest that marginal necrosis may easily occur owing to the shortness of the pedicle and the vulnerability of the colon to ischemia. This eventually leads to serious complications, such as fibrosis, perforation, infection, and sepsis. Neo-introitus reconstruction using a pudendal thigh island flap may be an effective way of completely resolving the resultant stricture and obstruction.

In 2018, Gupta et al. [6] reported a method for repairing neovaginal granulation tissue and stenosis using an extracellular porcine urinary bladder matrix (UBM). Similar to our case, complications in the rectosigmoid vagina of a transgender woman were repaired in a creative manner using a UBM. The granulation tissue was managed; however, neovaginal stenosis recurred after 6 months. The patient also required a dilation schedule of three times a day for several months. In comparison, the use of a pudendal thigh flap (as autologous tissue) eliminates the side effects of using a UBM and makes it possible to completely repair strictures functionally and cosmetically without using dilator devices or estrogen creams.

Although there are advantages of using autologous tissues (e.g., no recurrence and no need for dilator devices), further studies with more cases and longer follow-up durations should be conducted.

Nevertheless, our case is significant as it is the first case in South 
Korea wherein complete reconstruction of recurrent stricture and obstruction that developed after rectosigmoid vaginoplasty was performed using a pudendal thigh island flap.

A pudendal thigh flap can be considered as a way to effectively and safely release this type of stricture and to reconstruct the neointroitus of the rectosigmoid vagina without recurrence.

\section{NOTES}

\section{Conflict of interest}

No potential conflict of interest relevant to this article was reported.

\section{Ethical approval}

The study was approved by the Institutional Review Board of Hanyang University Seoul Hospital (IRB No. 2020-04-041) and performed in accordance with the principles of the Declaration of Helsinki.

\section{Patient consent}

The patient provided written informed consent for the publication and the use of her images.

\section{ORCID}

Tae Hyeon Lee Jung Soo Yoon https://orcid.org/0000-0002-2806-3885 https://orcid.org/0000-0003-2462-5702

Hee Chang Ahn

\section{REFERENCES}

1. Graziano K, Teitelbaum DH, Hirschl RB, et al. Vaginal reconstruction for ambiguous genitalia and congenital absence of the vagina: a $27-$ year experience. J Pediatr Surg 2002;37:955-60.

2. Kim SK, Park JH, Lee KC, et al. Long-term results in patients after rectosigmoid vaginoplasty. Plast Reconstr Surg 2003;112:143-51.

3. Dreher PC, Edwards D, Hager S, et al. Complications of the neovagina in male-to-female transgender surgery: a systematic review and metaanalysis with discussion of management. Clin Anat 2018;31:191-9.

4. van der Sluis WB, Bouman MB, Meijerink WJHJ, et al. Diversion neovaginitis after sigmoid vaginoplasty: endoscopic and clinical characteristics. Fertil Steril 2016;105:834-9.

5. Morrison SD, Satterwhite T, Grant DW, et al. Long-term outcomes of rectosigmoid neocolporrhaphy in male-to-female gender reassignment surgery. Plast Reconstr Surg 2015;136:386-94.

6. Gupta A, Francis S, Stewart R, et al. Repair of colonic neovaginal stenosis using a biological graft in a male-to-female transgender patient. Int Urogynecol J 2019;30:661-3. 